\title{
Combined effect of ADH1B rs1229984, rs2066702 and ADH1C rs1693482/ rs698 alleles on alcoholism and chronic liver diseases
}

\author{
Réka Tóth $^{\mathrm{a}, \mathrm{b}}$, Szilvia Fiatal ${ }^{\mathrm{a}, \mathrm{b}}$, Beáta Petrovski ${ }^{\mathrm{a}}$, Martin McKee ${ }^{\mathrm{c}}$ and Róza Ádány ${ }^{\mathrm{a}, \mathrm{b}, *}$ \\ ${ }^{a}$ University of Debrecen, Medical and Health Science Center, Faculty of Public Health, Department of Preventive \\ Medicine, Debrecen, Hungary \\ ${ }^{\mathrm{b}}$ Public Health Research Group of the Hungarian Academy of Sciences, Faculty of Public Health, Medical and \\ Health Science Centre, University of Debrecen, Debrecen, Hungary \\ ${ }^{\mathrm{c}}$ London School of Hygiene and Tropical Medicine, London, UK
}

\begin{abstract}
The aim of this study was to analyze the combined effect of the most frequent alcohol dehydrogenase polymorphisms (Arg48His and Arg370Cys in ADH1B, Arg272Gln and Ile350Val in ADH1C) on the alcohol use habits, alcohol dependence and chronic liver diseases in Hungary.

The study included men, aged 45-64 years. Altogether, 241 cases with chronic liver disease (CLD) and 666 randomly selected controls without CLD were analysed for all four polymorphisms. Associations between the polymorphisms, individually, and in combination, and excessive and problem drinking and CLD, were assessed using logistic regression.

In this study we have identified a novel mutation, called ADH1B Arg370His. The ADH1C Arg272Gln and Ile350Val showed almost complete linkage. The $272 \mathrm{Gln} / 350 \mathrm{Val}$ allele increased the risk of excessive and problem drinking in homozygous form $(\mathrm{OR}=1.582, p=0.035, \mathrm{CI}=1.034-2.421, \mathrm{OR}=1.780, p=0.016, \mathrm{CI}=1.113-2.848$, respectively $)$. The joint analysis showed that when combined with the wild type ADH1C Arg272/Ile350 allele, the ADH1B 48His is protective against CLD $(\mathrm{OR}=0.368, p=0.019, \mathrm{CI}=0.159-0.851)$.

The results obtained in the study help not only to clarify the effects of different ADH SNPs but to better understand how these polymorphisms modify each other's effects in the development of alcoholism and related diseases.
\end{abstract}

Keywords: Genetic epidemiology, genetic, case-control study, alcohol, chronic liver disease

\section{Introduction}

The burden of alcohol-related disease is especially high in the countries of Central and Eastern Europe [1]. In Hungary, the mortality from cirrhosis is more than 3 times higher than in Western European countries [2]. The risk of alcohol dependency (AD) and chronic liver

\footnotetext{
* Corresponding author: Roza Adany MD, Ph.D., D.Sc. University of Debrecen, Medical and Health Science Center, Faculty of Public Health, Department of Preventive Medicine, Kassai str. 26/b, Debrecen, Hungary. Tel.: +36 52460 190; Fax: +36 52417 267; E-mail: adany.roza@sph.unideb.hu.
}

diseases (CLDs), at an individual level, is affected by genetic factors [3].

Ingested alcohol is mainly metabolized in the liver, where the first step of oxidative degradation to acetaldehyde, is catalysed by the alcohol dehydrogenases (ADHs) mainly those in Class I [4]. Since the aldehyde dehydrogenase, catalysing the oxidation of acetaldehyde to acetate, has a low $\mathrm{K}_{m}$, the acetaldehyde produced is eliminated shortly after being formed [5]. Class I ADHs consist of $\alpha, \beta$ and $\gamma$ subunits, encoded by the genes ADH1A, ADH1B, and ADH1C. Single nucleotide polymorphisms (SNPs) have been described 
in both ADH1B and ADH1C that modify the enzymatic properties.

The ADH1B Arg48His allele (rs1229984) gives rise to the $\beta_{2}$ subunit with a 40 -fold higher $\mathrm{V}_{\max }$ in homozygous form than the $\beta_{1}$ subunit that is encoded by the wild allele. The ADH1B Arg370Cys (rs2066702) encodes the $\beta_{3}$ subunit. In its homodimer form, it has 30fold higher $\mathrm{V}_{\max }$ than $\beta_{1}[4,6]$. The wild type ADH1C $\left(\gamma_{1}\right.$ subunit $)$ contains arginine at $272 \mathrm{nd}$ and isoleucine at 350th position, while the ADH1C rs1693482/ rs698 variant, which encodes the $\gamma_{2}$ subunit, contains glutamine (rs1693482) and valine (rs698), respectively. The $\mathrm{V}_{\max }$ of $\gamma_{1}$ is 2-2.5 times higher than that of $\gamma_{2}$ [4]. These two SNPs are in high linkage disequilibrium [7]. Variants with higher activity $\left(\gamma_{1}, \beta_{2}\right.$ and $\left.\beta_{3}\right)$ are considered to give rise to acetaldehyde accumulation via faster ethanol degeneration, leading to adverse effects such as facial flushing, nausea and tachycardia [4,7]. These effects may deter further drinking, but if individuals persist with alcohol consumption, hepatotoxicity and other tissue damage may occur $[3,4,7]$.

Meta-analyses [8,9] have concluded that ADH1B Arg48 allele is associated with a significantly increased risk of alcoholism but is protective against liver disease only in Asian populations. ADH1C 272Gln/350Val allele seems to have little or no effect on alcoholism or liver disease in European populations. The difference between Asian and Caucasian populations may arise from the presence of different haplotypes [10].

The frequency of the polymorphic alleles varies among geographical regions. The ADH1B 48His allele is relatively rare in Caucasians with a frequency of 0 $10 \%$ [6,11-15] except in Russia where one study found it in $41 \%$ [16]. This allele is more frequent among Asian populations, where its frequency varies from 50 to 90 percent, depending on ethnicity $[15,17]$. The ADH1B 370Cys allele is mainly found among African Americans, with a prevalence of $10-35 \%$ but it is rare or absent in Caucasian and Asian populations $[6,10]$.

ADH1C 272Gln/350Val allele frequencies range from about 30 to $50 \%$ in Caucasians and strongly varies in Asian populations [6,11-14,17].

Although there has been considerable research among Caucasians on the effects of these SNPs in the recent years, only one study [18] analysed the combined effects of these polymorphisms, using only one outcome in each analysis, alcohol use or dependence. Moreover, almost all studies only investigated patients with alcoholic liver disease and alcoholic controls. In this study we describe not only the combined effect of the most frequent ADH polymorphisms both on CLDs,
AD and alcohol use but with including non-alcoholic cases and controls, we can also assess the possible effect of these polymorphisms on CLDs in moderate or rare drinkers. The results help not only to an improved understanding of the high prevalence of chronic liver disease in Hungary but it may contribute to better understanding of how these polymorphisms modify each other's effects in relation to alcohol consumption and liver disease.

\section{Materials and methods}

\subsection{Sample recruitment}

Subjects were recruited from 57 general practices in four Hungarian counties (Györ-Moson-Sopron, Zala, Hajdú-Bihar and Szabolcs-Szatmár-Bereg) participating in the General Practitioners' Morbidity Sentinel Stations Program [19]. The present study was restricted to men, aged 45-64 years. Cases were all subject identified as having chronic liver diseases while controls were selected at random from those with no diagnosed CLDs. The case definition, described previously [20], is the presence of at least two of the following pre-specified criteria based on physical examination: spider naevi; ascites; palmar and plantar erythema; jaundice; enlarged, firm liver with rounded or nodular edge; and at least one of the following laboratory findings: increased level of serum bilirubin, elevated aspartate transferase activity, elevated alanine transferase activity, elevated gamma-glutamyl-transpeptidase activity, elevated alkaline-phosphatase activity, or decreased serum albumin. These physical and laboratory examinations were conducted both on case and control patients. Where the findings of the tests were contradictory with the previous diagnosis, patient was transferred to the proper group according to the test results. Among cases, more than $65 \%$ were previously diagnosed by hepatologists, where the image examinations (ultrasound, CT, MRI) and liver biopsy were used to confirm the diagnosis. In case of the newly identified cases, the diagnostic tests were conducted also by specialists. In our study only screening, not diagnostic examinations were carried out just to define the presence or absence of liver diseases. Patients with hepatitis B or hepatitis $\mathrm{C}$ infection were excluded from the analysis. The patients were not screened for Cytomegalovirus or Epstein-Barr virus infections in the framework of the study; their serological status was classified later, during the detailed diagnostic examinations. The final 
study population contained 666 controls and 241 cases. The age distribution of controls was representative for the overall population $(p=0.424)$ and cases were significantly older than controls (55.17 years vs. 53.89 years, respectively, $p=0.0023$ ).

EDTA anti-coagulated blood samples were taken by general practitioners for routine biochemical tests and genotyping. Written informed consent was obtained from each patient. The study was approved by the Regional and Institutional Ethics Committee, Medical and Health Science Center, University of Debrecen. The study protocol conformed to the ethical guidelines of the 1975 Declaration of Helsinki.

\subsection{Questionnaire-based data collection}

Detailed information on alcohol consumption was gathered using a self-completed questionnaire, yielding the following variables.

Frequency of drinking, with seven possible outcomes: (i) has never drunk alcohol, (ii) didn't drink in the last twelve months, (iii) drinks less than once a month, (iv) drinks 1 to 3 times in a month, (v) 1 to 2 times in a week, (vi) 3 to 4 times in a week and (vii) at least 5 times in a week.

Summary drinking data, in 4 categories: (i) nondrinker; (ii) infrequent drinker, consuming alcohol less than 3 times per month, independently from the quantity of the consumed alcohol; (iii) moderate drinker, if consumption is at least weekly, the weekly total is 14 units or less, and the daily amount is never more than 5 units; (iv) heavy drinker, if more than 14 units are consumed weekly or if the amount more than 5 units is consumed on at least one day of a week ( 1 unit $=15 \mathrm{~g}$ pure ethanol).

CAGE score, from 0 to 4 , according to the number of positive answers on the CAGE questionnaire, a widely used tool to detect problem drinking [21].

$C A G E$ status is negative if the number of positive answers on the CAGE questionnaire is 0 or 1 , and positive if it is 2 or more.

Patterns of alcohol use and demographic, financial and educational characteristics in this sample have been published previously [22]. In the present study we analysed data only obtained on samples genotyped for all the 4 polymorphisms.

Education is categorised from 0 to 3, where 0 signifies an individual with 8 years of education or less, 1 signifies having attended secondary school without doing the school leaving exam, 2 and 3 signify completed secondary and higher education, respectively.
Financial status (self-assessed) has 3 possible values: 0 (bad/ very bad), 1 (adequate) and 2 (good/ very good). This has been found to have greater validity than questions on income or wealth in studies in this region [23].

Demographic, financial and educational characteristics and alcohol use habits of the study population are shown in Table 1.

\subsection{Genotyping}

DNA was isolated from leukocytes with the use of MagNA Pure LC DNA Isolation Kit - Large Volume (Roche Diagnostics GmbH, Mannheim, Germany) according to the manufacturer's instructions. Genotyping was performed on LightCycler real time PCR System (Roche Diagnostics GmbH, Mannheim, Germany) by melting curve analysis.

The genotyping for ADH1B Arg48His (rs1229984) was performed as described previously [22]. ADH1C Arg272Gln (rs1693482) and Ile350Val (rs698) polymorphisms were screened together in a duplex reaction, while ADH1B Arg370Cys (rs2066702) was analysed separately. Sequencing, where needed, was conducted by Biomi Ltd., Hungary, with one of the adequate PCR primers. The LightCycler reactions were performed with LightCycler DNA Master HybProbe (Roche Diagnostics, GmbH, Mannheim, Germany, Cat. No. 12015 102 001), according the manufacturers' instructions. The probes applied in the melting curve analysis were used in $0.2 \mu \mathrm{mol} / 1$ concentrations, while the primers were in $0.5 \mu \mathrm{mol} / 1$ concentrations. The sequence of the primers, probes and the reaction conditions are shown in the Table 2.

\subsection{Statistical analyses on samples used for:}

\subsubsection{Single SNP analysis}

Logistic regression calculating odds ratios (ORs) was applied to test the association between each genotype and the outcome variables, presence of chronic liver disease, frequency of drinking, summary drinking data, number of positive CAGE answers given and CAGE status. Results were adjusted for age, financial and educational status. Where variables were non-binary, ordered logistic regression was used which is appropriate to estimate the relationship between an ordinal dependent variable and a set of independent variables.

With the ADH1B Arg48His and the newly identified ADH1B Arg370His, the dominant model was used by virtue of the low number of homozygous mutant samples while for ADH1C Arg272GIn and Ile350Val the additive model proved the best fit. 
Table 1

Demographic, educational, financial characteristics and alcohol use in the study population

\begin{tabular}{|c|c|c|c|}
\hline & & Controls & Cases \\
\hline Age & Mean \pm SD & $53.89 \pm 5.59$ & $55.17 \pm 5.49$ \\
\hline \multicolumn{4}{|c|}{ Education } \\
\hline \multirow[t]{6}{*}{ Data } & & 659 & 239 \\
\hline & & $\%$ & $\%$ \\
\hline & 8 years of education & 21.85 & 28.87 \\
\hline & Secondary school without exam & 42.64 & 49.37 \\
\hline & Secondary school & 21.70 & 17.15 \\
\hline & Higher education & 13.81 & 4.60 \\
\hline \multicolumn{4}{|c|}{ Financial status } \\
\hline \multirow[t]{5}{*}{ Data } & & 662 & 241 \\
\hline & & $\%$ & $\%$ \\
\hline & Bad/very bad & 16.92 & 22.82 \\
\hline & Adequate & 68.13 & 67.22 \\
\hline & Good/very good & 14.95 & 9.96 \\
\hline \multicolumn{4}{|c|}{ Frequency of drinking } \\
\hline \multirow[t]{9}{*}{ Data } & & 644 & 236 \\
\hline & & $\%$ & $\%$ \\
\hline & Never & 13.66 & 7.20 \\
\hline & Did not drink in the last 12 month & 2.80 & 3.39 \\
\hline & Less than 1 time in a month & 7.14 & 2.54 \\
\hline & $1-3$ times in a month & 13.04 & 3.39 \\
\hline & $1-2$ times in a week & 22.20 & 6.78 \\
\hline & 3-4 times in a week & 15.84 & 17.80 \\
\hline & At least 5 times a week & 25.31 & 58.90 \\
\hline \multicolumn{4}{|c|}{ Summarised drinking data } \\
\hline \multirow[t]{6}{*}{ Data } & & 625 & 212 \\
\hline & & $\%$ & $\%$ \\
\hline & Non-drinker & 20.16 & 13.68 \\
\hline & Infrequent drinker & 11.20 & 4.72 \\
\hline & Moderate drinker & 21.44 & 13.68 \\
\hline & Heavy drinker & 47.20 & 67.92 \\
\hline \multicolumn{4}{|c|}{ Number of positive CAGE answers given } \\
\hline \multirow[t]{7}{*}{ Data } & & 538 & 201 \\
\hline & & $\%$ & $\%$ \\
\hline & 0 & 55.20 & 15.42 \\
\hline & 1 & 18.03 & 18.41 \\
\hline & 2 & 10.59 & 19.40 \\
\hline & 3 & 9.67 & 27.36 \\
\hline & 4 & 6.51 & 19.40 \\
\hline \multicolumn{4}{|c|}{ CAGE status } \\
\hline \multirow[t]{4}{*}{ Data } & & 538 & 201 \\
\hline & & $\%$ & $\%$ \\
\hline & Negative & 73.23 & 33.83 \\
\hline & Positive & 26.77 & 66.17 \\
\hline
\end{tabular}

\subsubsection{Linkage analysis}

To map the linkage between the SNPs, LD, D' and $\mathrm{r}^{2}$ values were calculated using Haploview Software [24].

\subsubsection{Multivariate SNP analysis}

To detect the combined effect of the three main polymorphisms (48His, $272 \mathrm{Gln}, 350 \mathrm{Val}$ ) and the possible epistasis between them, they were entered into a multivariate logistic regression model. Altogether, five groups were formed according to the genotype status of the polymorphisms investigated: the sam- ples in the first group (named wild/wild) were homozygous wild for all three mutations and served as reference group; in the second (wild/heterozygous) they were wild for $\mathrm{Arg} 48 \mathrm{His}$ and heterozygous for Arg272Gln and Ile350Val; the members of the third group (wild/mutant) were also wild for Arg48His, but homozygous mutant for Arg272Gln/ Ile350Val; the fourth group (heterozygous/wild) contained carriers for 48His and wild samples for Arg272Gln/ Ile350Val, while the fifth group (heterozygous/heterozygous) was formed from samples carrying the $48 \mathrm{His}$ allele and were heterozygous for Arg272Gln/ Ile350Val (Table 3). 


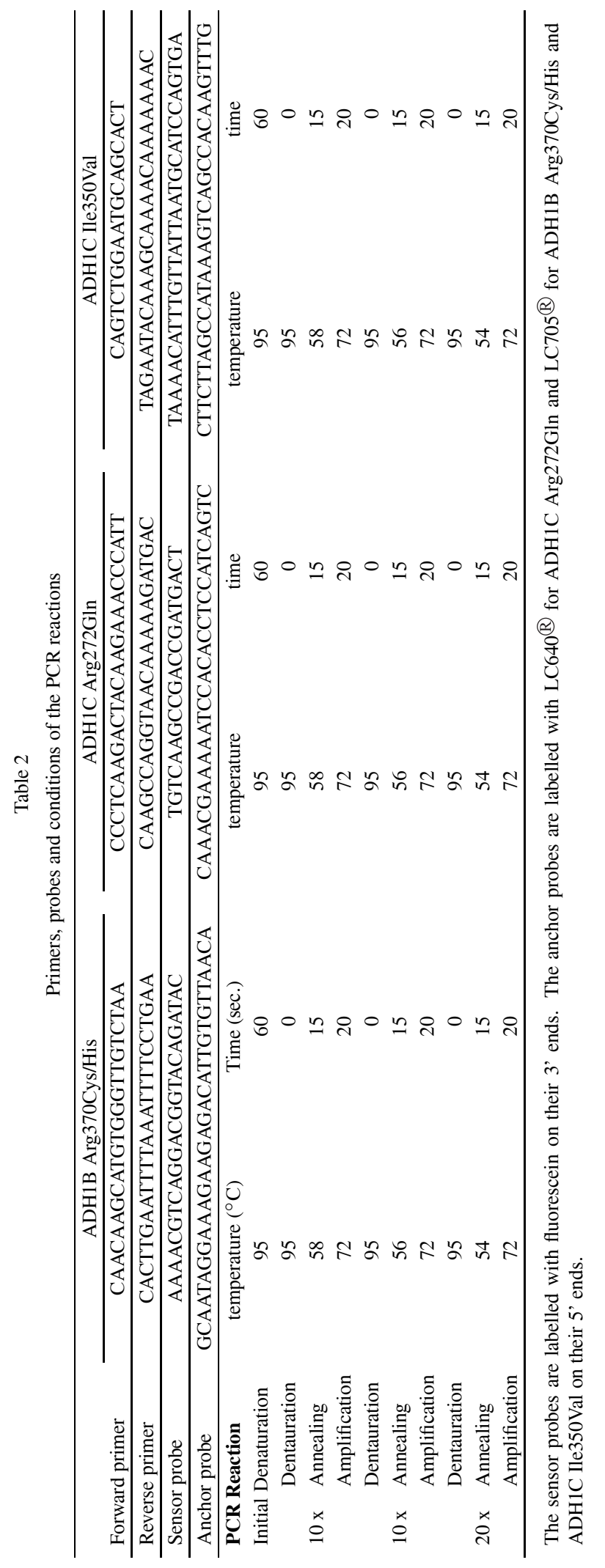


Table 3

Genotypes of polymorphisms in groups used for multivariate SNP analysis

\begin{tabular}{llll}
\hline & ADH1B Arg48His & ADH1C Arg272Gln & ADH1C Ile350Val \\
\hline $\begin{array}{l}\text { First group } \\
\text { (wild/wild) }\end{array}$ & homozygous wild & homozygous wild & homozygous wild \\
$\begin{array}{l}\text { Second group } \\
\text { (wild/heterozygous) }\end{array}$ & homozygous wild & heterozygous & heterozygous \\
$\begin{array}{l}\text { Third group } \\
\text { (wild/mutant) }\end{array}$ & homozygous wild & homozygous mutant & homozygous mutant \\
$\begin{array}{l}\text { Fourth group } \\
\text { (heterozygous/wild) }\end{array}$ & heterozygous & homozygous wild & homozygous wild \\
$\begin{array}{l}\text { Fifth group } \\
\text { (heterozygous/heterozygous) }\end{array}$ & heterozygous & heterozygous & heterozygous \\
\hline * served as reference type. & & & \\
\hline
\end{tabular}

Any other combinations of these mutations and the other polymorphisms involved in this study were present in such a low number as to generate potentially ambiguous results, therefore they were excluded from the analyses. Logistic regression was used to measure the association between these genotype combinations and CLDs or alcohol consumption habits. Results are expressed with both $95 \%$ confidence intervals and $p$ values at the $5 \%$ significance level. Data were analysed using STATA 10.0 statistical software (StataCorp LP, Texas, USA).

\section{Results}

Data on alcohol use and the demographic, financial and educational characteristics of the study population have been shown previously in Table 1 .

\subsection{Single SNP analysis}

\subsubsection{Identification of a new SNP variant}

During the analysis of the ADH1B Arg370Cys (rs2066702) mutation an unexpected polymorphism was detected, causing approximately $2^{\circ} \mathrm{C}$ lowering in the melting temperature compared to the wild type (the probe was specific for the mutant type). Sequencing revealed this to be a recently identified gene variant in which the same amino acid is affected but with substitution of histidine instead of cysteine. This variant is Arg370His (rs75967634), caused by a G to A substitution in the 1193rd mRNA position. Figure 1 shows the results of the sequencing. Although this mutation has been submitted recently to PubMed's SNP database, no data are yet available on its frequency or its effect, so it was included in the further analysis. It was present in $1.98 \%$ of the study population.

\subsubsection{Analysing the included SNPs}

The allele frequencies and genotype frequencies of ADH polymorphic regions studied are shown in Table 4. All mutations examined were in Hardy-Weinberg equilibrium in both cases and controls.

Odds ratios were calculated by using data obtained on samples analysed for all 4 polymorphisms (Table 5).

Carriage of the ADH1B 48His allele was associated with significantly lower odds ratio for drinking frequency, the number of positive answers on CAGE assessment, and positive CAGE status. There was a significant association between ADH1B 48His and CLDs, but it disappeared after adjusting for CAGE status and scores $(\mathrm{OR}=0.61, p=0.101 ; \mathrm{OR}=0.63, p=0.123$, respectively).

The ADH1B 370His allele was not associated with any of the examined outcomes.

The ADH1B Arg370Cys was excluded from further analysis as a consequence of its low frequency $(<1 \%)$.

The frequency of ADH1C 272Gln was almost the same as ADH1C 350Val and there were only two samples with different genotypes for these two mutations (Table 4) therefore they were excluded from further analysis and the effect of these polymorphisms were calculated together in the single SNP analysis. These two mutations showed no association with CLDs, but they were associated with increased odds ratio for frequency of drinking, summary drinking data and CAGE status. However, these associations were only significant when the mutations were homozygous.

The results of the single SNP analyses can be seen in Table 5.

\subsection{Linkage analysis}

The results of the linkage analysis and the frequency of the main haplotypes are shown in Fig. 2. As expected, there is a very high linkage between the two 
170
$: A C G T C A G G A C G G T A Z H G A T A C T$ G $\triangle A A T A G G A A A C$

A

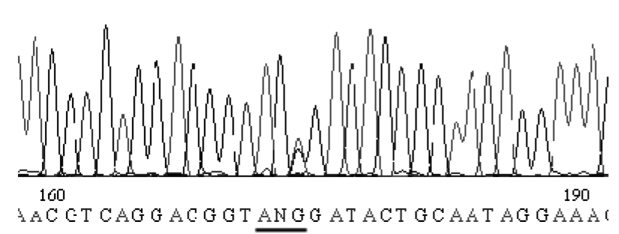

B

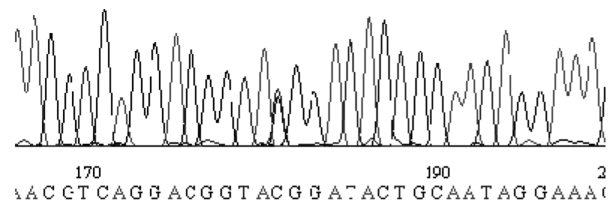

C

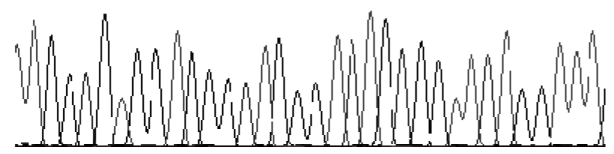

$\mathrm{ACA} \rightarrow \mathrm{TGT} \rightarrow \mathrm{Cys}$

rs2066702

$\mathrm{ATG} \longrightarrow \mathrm{CAT} \longrightarrow \mathrm{His}$

rs75967634

$\mathrm{ACG} \longrightarrow \mathrm{CGT} \longrightarrow \mathrm{Arg}$

wild type allele

Fig. 1. The results of the sequencing of the ADH1B Arg370Cys/Arg370His polymorphism. Part A shows the coding sequence of a sample heterozygous for $370 \mathrm{Cys}$, while on part B, the sequence coding a heterozygous 370His variant can be seen. Part C represents the wild type (homozygous for Arg370).

ADH1C mutations. The high D' combined with the low $\mathrm{r}^{2}$ values in case of ADH1C 272Gln and 350Val versus $\mathrm{ADH} 1 \mathrm{~B} 48 \mathrm{His}$ indicate that the combination of wild-wild-mutant and mutant-mutant-wild alleles is more frequent in the population than could be expected from the allele frequencies themselves.

\subsection{Multivariate SNP analysis}

The results of the multivariate analysis are shown in Table 6.

For drinking habits, only the frequency of drinking was significantly associated with the heterozygous/wild group, with an odds ratio of $0.550(p=0.011, \mathrm{CI}=$ 0.346-0.874).

There was no significant difference for either the summary drinking data or the number of positive CAGE answers.

Both wild/heterozygous and wild/mutant groups resulted in higher odds ratios for positive CAGE status $(\mathrm{OR}=1.540, p=0.024, \mathrm{CI}=1.058-2.241 ; \mathrm{OR}=$ $1.859, p=0.014, \mathrm{CI}=1.136-3.042)$. Interestingly, neither the heterozygous/wild, nor the heterozygous/heterozygous showed difference for CAGE status.

Significant association with CLDs was found only in case of groups containing the mutant allele for
Arg48His. Both appeared to be protective, with odds ratios of 0.398 (heterozygous/wild, $p=0.010, \mathrm{CI}=$ $0.197-0.806$ ) and 0.461 (heterozygous/heterozygous, $p=0.043$, CI $=0.218-0.975)$.

To clarify whether these combinations act directly on the risk of chronic liver disease or via drinking habits (i.e. CAGE status), these outcome variables were entered together into a logistic regression model to assess their effect when controlling for the other outcome variable. When CAGE status was used as outcome and the presence of chronic liver disease was adjusted for, the association with wild/heterozygous and wild/mutant groups remained significant $(\mathrm{OR}=1.756$, $p=0.006, \mathrm{CI}=1.171-2.633 ; \mathrm{OR}=2.204, p=0.004$, $\mathrm{CI}=1.287-3.774)$. When CLD was used as a dependent variable and results were adjusted for CAGE status, only the effect of the heterozygous/wild group on CLD remained significant $(\mathrm{OR}=0.368, p=0.019$, $\mathrm{CI}=0.159-0.851)$.

To assess the possible effects of these combinations on CLDs among non-alcoholics, we entered them into a logistic regression model restricted only to CAGE negative cases and controls. Only the heterozygous/wild group showed significant association with CLDs $(\mathrm{OR}=0.116, p=0.039, \mathrm{CI}=0.015-0.900)$. 
Table 4

Genotype and allele frequencies of the polymorphisms studied

\begin{tabular}{|c|c|c|c|c|c|c|c|c|c|c|}
\hline & \multicolumn{4}{|c|}{ Allele frequency } & \multicolumn{4}{|c|}{ Genotype frequency } & & \\
\hline & \multicolumn{2}{|c|}{ wt } & \multicolumn{2}{|c|}{$\mathrm{mt}$} & \multicolumn{2}{|c|}{ wt/wt } & \multicolumn{2}{|c|}{$\mathrm{wt} / \mathrm{mt}$} & \multicolumn{2}{|c|}{$\mathrm{mt} / \mathrm{mt}$} \\
\hline & Cases & Cont. & Cases & Cont. & Cases & Cont. & Cases & Cont. & Cases & Cont. \\
\hline $\begin{array}{l}\text { ADH1B Arg48His } \\
(\text { rs1229984) }\end{array}$ & 0.956 & 0.914 & 0.044 & 0.086 & 0.913 & 0.832 & 0.087 & 0.165 & 0.000 & 0.003 \\
\hline $\begin{array}{l}\text { ADH1B Arg370His } \\
\text { (rs75967634) }\end{array}$ & 0.990 & 0.990 & 0.010 & 0.010 & 0.979 & 0.981 & 0.021 & 0.020 & 0.000 & 0.000 \\
\hline $\begin{array}{l}\text { ADH1B Arg370Cys } \\
\text { (rs2066702) }\end{array}$ & 1.000 & 0.999 & 0.000 & 0.001 & 1.000 & 0.997 & 0.000 & 0.003 & 0.000 & 0.000 \\
\hline $\begin{array}{l}\text { ADH1C Arg272Gln } \\
\text { (rs1693482) / Ile350Val } \\
(\text { rs698) }\end{array}$ & 0.613 & 0.613 & 0.387 & 0.387 & 0.375 & 0.367 & 0.475 & 0.492 & 0.150 & 0.141 \\
\hline
\end{tabular}

$\mathrm{wt}=$ wild type, $\mathrm{mt}=$ mutant type, cont. $=$ controls.

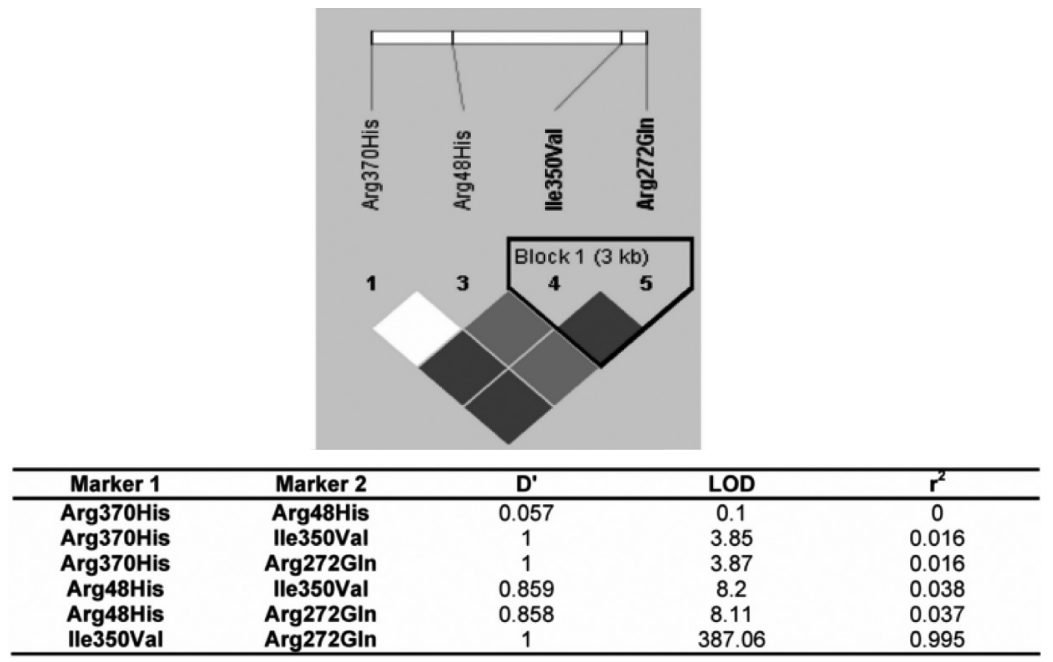

Fig. 2. The results of the linkage analysis. The upper part shows the LD plot, where the intensity of the colour indicates the D'/LOD values. The lower part contains the D', LOD and $\mathrm{r}^{2}$ values between the SNPs.

\section{Discussion}

Although there have been recent declines, death rates from chronic liver diseases and cirrhosis remain much higher in the majority of the Central-Eastern European countries - among them Hungary - than in the countries of western Europe. In previous years, the potential role of the quality of alcohol consumed in this region has been examined $[25,26]$ but potential genetic factors have received less attention. Although research has been conducted in other European countries [12-14,16, 18,27-32], the combined effects of the main polymorphisms remain to be clarified. In a previous study [22], we have taken a first step by mapping the prevalence of ADH1B Arg48His in Hungary. In this study, our goal was not only to wider this investigation to other, also important SNPs, but to examine their joint impacts on chronic liver diseases, alcohol dependence and al- cohol drinking habits not only among alcoholic cases and controls but among moderate and rare drinkers as well. The results of this complex analysis begin to offer insights into the genetic background of alcohol consumption and alcohol-related diseases not only among Hungarians but among other Caucasian populations.

An interesting, unexpected finding of our study was the identification of a novel mutation, the ADH1B Arg370His (rs75967634), and assessed its frequency. This polymorphic sequence has already been submitted into the PubMed's SNP database, but without any information on the population studied, its frequency, or possible effects. Because of its close proximity to ADH1B Arg370Cys, it cannot be detected with either the original hybridization probe assay [33] or with newer methods such as Illumina GoldenGate or Sequenom MassArray techniques and RFLP. A highly specific melting point analysis as used in this study is required to detect 

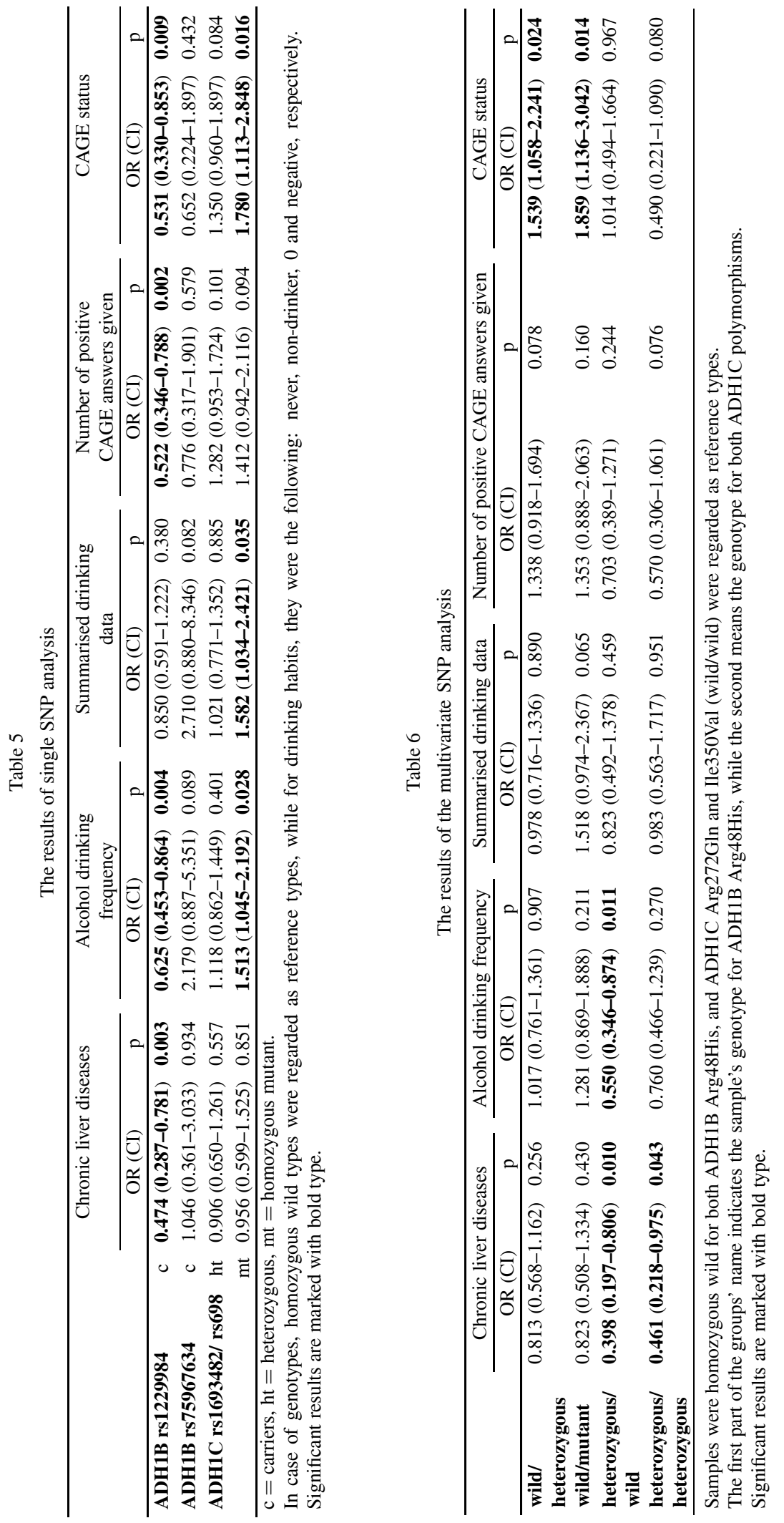
it. Although we did not find any significant association with alcohol drinking habits and liver diseases, there may be possible associations that could not be identified in the present study because of its low prevalence. Consequently, the effect of this SNP needs further investigation, including studies in other populations.

The results for ADH1B Arg48His in the samples used in this study show concordance with the findings of our previous study [22], as was expected.

The two main polymorphisms of ADH1C, the Arg272Gln and Ile350Val have an almost complete linkage between each other, as it was expected from previous studies [34]. However, the results regarding the effect of these mutations are not so obvious among Caucasians. Although previous meta-analyses and other studies $[8,9,13,14]$ did not find an association between these alleles and alcoholism or liver disease, some researchers have described associations with alcohol drinking habits and/or alcoholism among Europeans [18,30,32]. However, while our results confirm the lack of association with CLDs, we did find a significantly increased OR for problem drinking (i.e. positive CAGE status) and heavy drinking in ADH1C $272 \mathrm{Gln} / 350$ Val homozygous patients. This ambiguity may be due to the differences in categorizing drinking habits in different studies and the relatively minor effect of this genotype. Our results of the linkage disequilibrium completely concur with previous studies $[11,14$, 18], which showed that ADH1B 48His allele is associated with the Arg272/Ile350 ADH1C allele.

To clarify the independent effect of the main polymorphisms of $\mathrm{ADH} 1 \mathrm{~B}$ and $\mathrm{ADH} 1 \mathrm{C}$, a multivariate analysis was conducted. When CLD and alcohol use habits were used as an outcome variable without controlling for each other, the results were what were expected on the basis of the single SNP analysis, except that being heterozygous for the ADH1B 48His allele did not reduce the odds of problem drinking. This can be due that this allele has an adverse effect on CAGE status in cases and controls, e.g. in cases it did not proved to be protective against CAGE positivism ( $p=$ 0.371). Combined with the relatively low number of subjects in these categories it can result in the lack of significant association.

Interestingly, when CAGE status was used as an outcome variable and adjustment was made for the presence of CLD, only the association with homozygous ADH1B Arg48 status remained significant. When CLD was the outcome variable and CAGE status was controlled for, the association with carriage of ADH1B 48His and homozygous for ADH1C Arg272/Ile350 re- mained significant. These results suggest that it is reasonable to suppose that the polymorphisms of ADH1C have a direct impact only on drinking habits. The outcome of the single SNP analyses also supports this hypothesis. On the contrary, while the ADH1B 48His allele seemed to impact only on drinking habits on the basis of previous individual analyses [22], these results suggest that the absence of the controversial ADH1C 272Gln/350Val alleles are significantly protective against chronic liver disease. This is also supported by the significantly protective effect against CLDs of the group that was heterozygous for ADH1B 48His allele and homozygous for ADH1C Arg272/Ile350 alleles in non-alcoholics (i.e. negative CAGE status). However, this hypothesis needs to be confirmed by enzymatic tests to assess enzyme activity and the consequent alcohol elimination rate in subjects with these allelic combinations or by other case-control studies involving non-alcoholic cases and controls as well.

It is important to recognize that our study may underestimate the real effect of the genotypes studied as we have no data about individuals who have stopped drinking after being diagnosed with liver disease. Nevertheless, this work represents an important step in determining the genetic background of alcoholism and chronic liver diseases in Caucasians as it is one of the most complex studies conducted in Europe, involving a remarkably high number of cases and controls and yielding novel and interesting findings, including new interactions between ADH SNPs, alcohol dependence and CLDs.

\section{Acknowledgements}

The design and synthesis of the labelled detection probes and unlabelled PCR primers are highly acknowledged to TIB MolBiol Co. (Berlin, Germany). The efforts of the general practitioners to collect data and blood samples, Zsuzsa Pocsai, Laszlo Kardos and Gyorgy Szeles to the involvement in the early phase of the study, as well as the contribution of the public health offices to the quality assurance of the survey are acknowledged.

This work was supported by the Hungarian Academy of Sciences [2006TKI247] and the Jedlik Ányos Program of "Potentials of preventing diseases of major public health importance" [NKFP1-00003/2005]. 


\section{References}

[1] J. Rehm et al., Alcohol accounts for a high proportion of premature mortality in central and eastern Europe, Int J Epidemiol 36(2) (2007), 458-467.

[2] W.A. Zatonski et al., Liver cirrhosis mortality in Europe, with special attention to Central and Eastern Europe, Eur Addict Res 16(4) (2010), 193-201.

[3] F. Stickel and C.H. Osterreicher, The role of genetic polymorphisms in alcoholic liver disease, Alcohol Alcohol 41(3) (2006), 209-224

[4] D.W. Crabb et al., Overview of the role of alcohol dehydrogenase and aldehyde dehydrogenase and their variants in the genesis of alcohol-related pathology, Proc Nutr Soc 63(1) (2004), 49-63.

[5] S. Gemma, S. Vichi and E. Testai, Individual susceptibility and alcohol effects:biochemical and genetic aspects, Ann Ist Super Sanita 42(1) (2006), 8-16.

[6] H.J. Edenberg et al., Association of alcohol dehydrogenase genes with alcohol dependence: a comprehensive analysis, Hum Mol Genet 15(9) (2006), 1539-1549.

[7] H.J. Edenberg, The genetics of alcohol metabolism: role of alcohol dehydrogenase and aldehyde dehydrogenase variants, Alcohol Res Health 30(1) (2007), 5-13.

[8] E. Zintzaras et al., Do alcohol-metabolizing enzyme gene polymorphisms increase the risk of alcoholism and alcoholic liver disease? Hepatology 43(2) (2006), 352-361.

[9] J.B. Whitfield, Meta-analysis of the effects of alcohol dehydrogenase genotype on alcohol dependence and alcoholic liver disease, Alcohol Alcohol 32(5) (1997), 613-619.

[10] M.V. Osier et al., A global perspective on genetic variation at the ADH genes reveals unusual patterns of linkage disequilibrium and diversity, Am J Hum Genet 71(1) (2002), 84-99.

[11] S. Macgregor et al., Associations of ADH and ALDH2 gene variation with self report alcohol reactions, consumption and dependence: an integrated analysis, Hum Mol Genet 18(3) (2009), 580-593.

[12] A. Lorenzo et al., Polymorphisms of alcohol-metabolizing enzymes and the risk for alcoholism and alcoholic liver disease in Caucasian Spanish women, Drug Alcohol Depend 84(2) (2006), 195-200.

[13] F. Vidal et al., Genetic polymorphisms of $\mathrm{ADH} 2, \mathrm{ADH} 3$, CYP4502E1 Dra-I and Pst-I, and ALDH2 in Spanish men: lack of association with alcoholism and alcoholic liver disease, J Hepatol 41(5) (2004), 744-750.

[14] E. Borras et al., Genetic polymorphism of alcohol dehydrogenase in europeans: the $\mathrm{ADH} 2 * 2$ allele decreases the risk for alcoholism and is associated with $\mathrm{ADH} 3 * 1$, Hepatology 31(4) (2000), 984-989.

[15] H.W. Goedde et al., Distribution of ADH2 and ALDH2 genotypes in different populations, Hum Genet 88(3) (1992), 344 346.

[16] P.P. Ogurtsov et al., Alcohol dehydrogenase ADH2-1 and ADH2-2 allelic isoforms in the Russian population correlate with type of alcoholic disease, Addict Biol 6(4) (2001), 377383.

[17] L. Ma et al., Polymorphism study of seven SNPs at ADH genes in 15 Chinese populations, Hereditas 142(2005) (2005), $103-111$.
[18] J.S. Tolstrup et al., Alcoholism and alcohol drinking habits predicted from alcohol dehydrogenase genes, Pharmacogenomics J 8(3) (2008), 220-227.

[19] G. Szeles et al., [Establishment and preliminary evaluation of the General Practitioners' Morbidity Sentinel Stations Program in Hungary. Prevalence of hypertension, diabetes mellitus and liver cirrhosis], Orv Hetil 144(31) (2003), 1521-1529.

[20] G. Szeles et al., A preliminary evaluation of a health monitoring programme in Hungary, Eur J Public Health 15(1) (2005), 26-32.

[21] J.A. Ewing, Detecting alcoholism. The CAGE questionnaire, Jama 252(14) (1984), 1905-1907.

[22] R. Toth et al., ADH1B*2 allele is protective against alcoholism but not chronic liver disease in the Hungarian population, Addiction 105(5) (2010), 891-896.

[23] D.C. Balabanova and M. McKee, Self-reported health in Bulgaria: levels and determinants, Scand J Public Health 30(4) (2002), 306-312.

[24] J.C. Barrett et al., Haploview: analysis and visualization of LD and haplotype maps, Bioinformatics 21(2) (2005), 263-265.

[25] D.W. Lachenmeier, B. Sarsh and J. Rehm, The composition of alcohol products from markets in Lithuania and Hungary, and potential health consequences: a pilot study, Alcohol Alcohol 44(1) (2009), 93-102.

[26] S. Szucs et al., Could the high level of cirrhosis in central and eastern Europe be due partly to the quality of alcohol consumed? An exploratory investigation, Addiction 100(4) (2005), 536-542.

[27] H. Cichoz-Lach et al., Alcohol dehydrogenase and aldehyde dehydrogenase gene polymorphism in alcohol liver cirrhosis and alcohol chronic pancreatitis among Polish individuals, Scand J Gastroenterol 42(4) (2007), 493-498.

[28] J.H. van Beek et al., Associations between ADH gene variants and alcohol phenotypes in Dutch adults, Twin Res Hum Genet 13(1) (2010), 30-42.

[29] C. Coutelle et al., Population distribution of alcohol dehydrogenase class I in France: comparison with other populations, and distribution with respect to gender and age. The French Group for Research on Alcohol and Liver, Alcohol Alcohol 33(2) (1998), 173-183.

[30] H. Cichoz-Lach et al., Genetic polymorphism of alcoholmetabolizing enzyme and alcohol dependence in Polish men, Braz J Med Biol Res 43(3) (2010), 257-261.

[31] P.H. Kuo et al., Association of ADH and ALDH genes with alcohol dependence in the Irish Affected Sib Pair Study of alcohol dependence (IASPSAD) sample, Alcohol Clin Exp Res 32(5) (2008), 785-795.

[32] A. Linneberg et al., Genetic determinants of both ethanol and acetaldehyde metabolism influence alcohol hypersensitivity and drinking behaviour among Scandinavians, Clin Exp Allergy 40(1) (2010), 123-130.

[33] Y.L. Xu et al., Genotyping of human alcohol dehydrogenases at the ADH2 and ADH3 loci following DNA sequence amplification, Genomics 2(3) (1988), 209-214.

[34] L.L. Husemoen et al., The association of ADH and ALDH gene variants with alcohol drinking habits and cardiovascular disease risk factors, Alcohol Clin Exp Res 32(11) (2008), 1984-1991. 


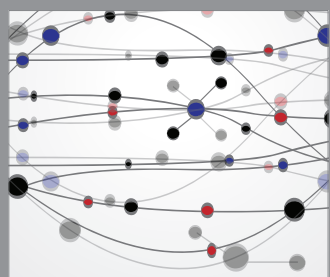

The Scientific World Journal
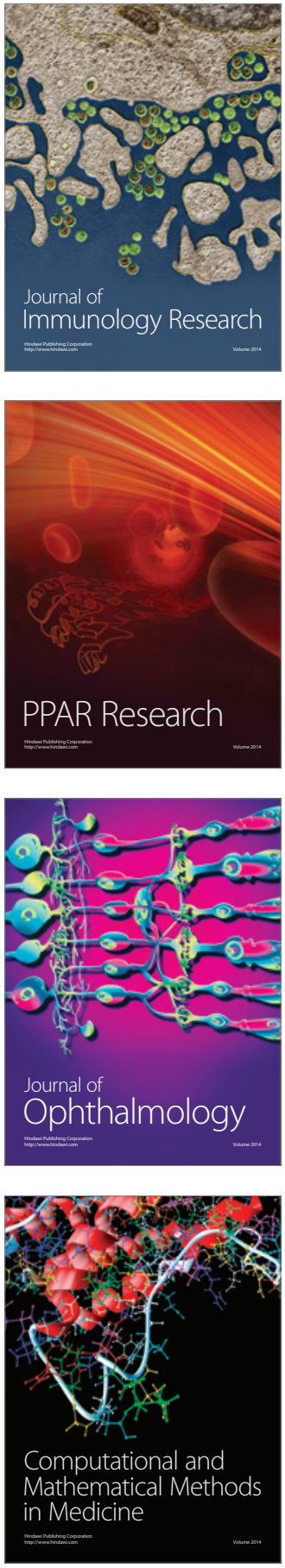

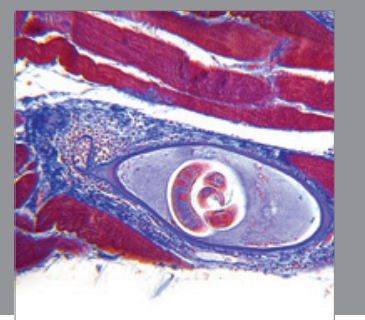

Gastroenterology

Research and Practice
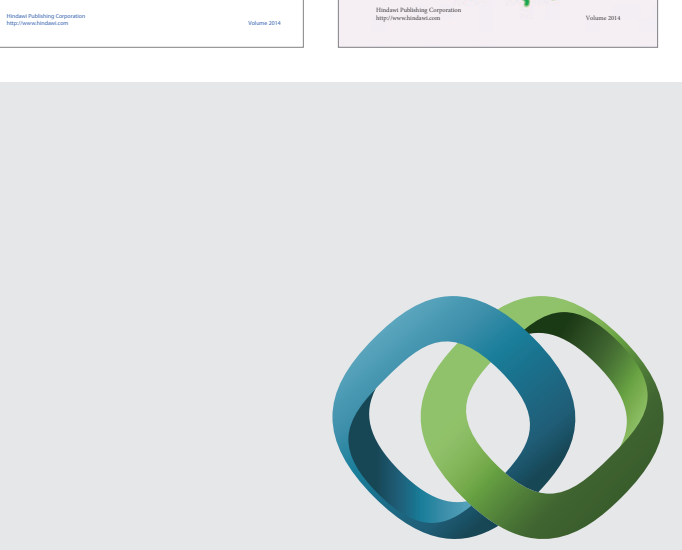

\section{Hindawi}

Submit your manuscripts at

http://www.hindawi.com
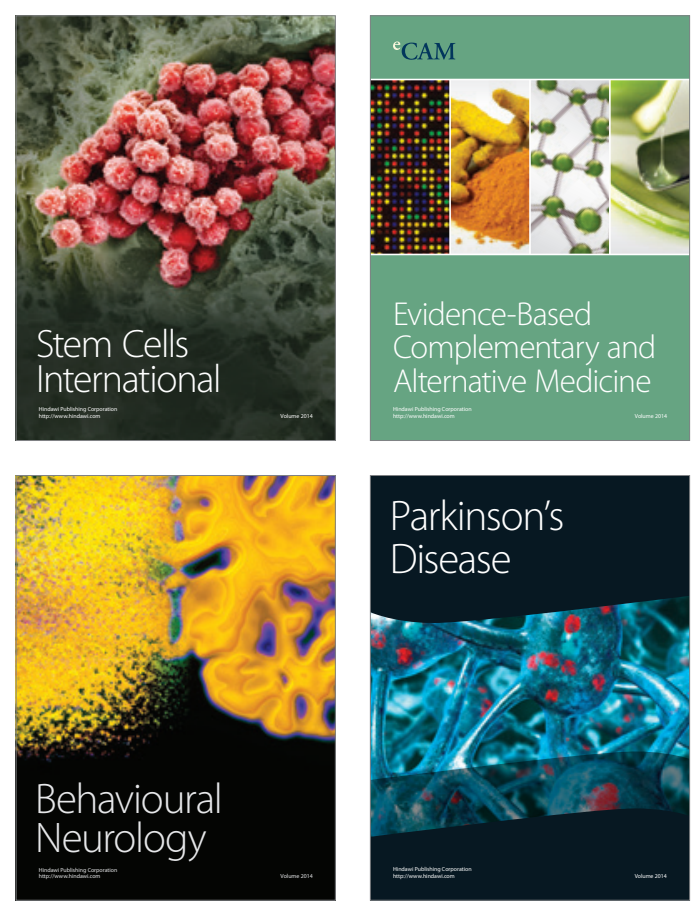

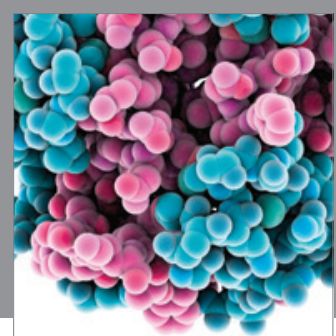

Journal of
Diabetes Research

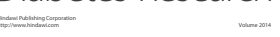

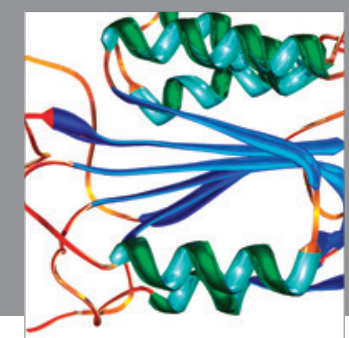

Disease Markers
